\title{
The Effect on Model Identifiability of Allowing Different Relocation Rates for Live and Dead Animals in the Combined Analysis of Telemetry and Recapture Data
}

\author{
Marlina D. NAsution, Cavell Brownie, \\ Kenneth H. Pollock, and Roger A. Powell
}

\begin{abstract}
Models are described for the joint analysis of live-trapping and radio telemetry data from a study on black bears (Ursus americanus) in which all animals received ear tags and a subset also received radio tags. Concerns about bias in survival estimates led to investigation of identifiability and estimator precision for a series of models that allowed different telemetry relocation rates for living and dead animals, in addition to emigrationand seasonal variation in survival. Identifiability was determined by showing that the expected information matrix was nonsingular. Models with fidelity constant across time, and with the same degree of time specificity for survival rates and relocation rates for dead animals, were determined to be nonidentifiable. More general models, with a greater degree of time specificity for survival rates, were near-singular, and estimators under these near-singular models had poor precision. Analysis of data from the study on black bears illustrated that estimates of survival have poor precision when relocation rates are estimated separately for live and dead animals. It is recommended that the effort expended to relocate both living and dead animals be consistently high in each telemetry survey, so that relocation rates will be high and constant across time and mortality status.
\end{abstract}

Key Words: Capture-recapture;Near-singularity;Overparameterization;Radio telemetry; Unequal catchability; Wildlife studies.

\section{INTRODUCTION}

Capture-recapture studies that use more than one type of tag and more than one recapture method are becoming increasingly common. Using more than one type of tag means

Marlina D. Nasution is Biostatistician, Family Health International, P.O. Box 13950, Research Triangle Park, NC 27709(E-mail: mnasution @ fhi.org).Cavell Brownie is Professor, and Kenneth H. Pollock is Professor, Department of Statistics, North Carolina State University, Raleigh, NC 27695-8203. Roger A. Powell is Professor, Department of Zoology, North Carolina State University, Raleigh, NC 27695-7617.

(C)2004 American Statistical Association and the International Biometric Society Journal of Agricultural, Biological, and Environmental Statistics, Volume 9, Number 1, Pages 27-41 DOI: $10.1198 / 1085711043181$ 
that survival estimates will be more precise, and may allow more parameters to be estimated. A possible disadvantage of using more than one type of tag is that constructing a model and likelihood for an efficient analysis of the combined datasets can be considerably more complex than for data from a single recapture method.

An important study design that uses two types of tags involves augmenting a traditional tagging study by placing radios on a subset of the marked animals. Recapture efforts are often carried out annually, whereas telemetry surveys to relocate the animals with radio tags are conducted more frequently. Using radio tags makes it possible to estimate withinyear variation in survival, and to estimate separately fidelity and survival probabilities. In contrast, only the product of annual fidelity and survival probabilities can be estimated from the traditional recapture data. Two studies that combined telemetry and traditional recaptures are a resighting study on snail kites (Rhostrhamus sociabilis) in which a subset of the leg-banded birds also received radio tags (Bennetts et al. 1999), and a live-trapping study on black bears (Ursus americanus), some of which were fitted with radio collars in addition to ear tags (Sorensen and Powell 1998). Nasution, Brownie, and Pollock (2001) presented the likelihood for the combined analysis of the telemetry and resighting data from the snail kite study, but only separate analyses of the telemetry and capture-recapture data have been reported for the study on black bears. As sample sizes were necessarily small in the study on black bears, it is of interest to determine whether any additional information can be obtained by a combined analysis of the telemetry and recapture data.

Nasution et al. (2001) noted the complexity of constructing a model for the data from a study with two types of tags and recapture methods. The relationship between the biological processes of survival and movement and the timing and location of the sampling procedures must be considered, and many different types of parameters may be needed to describe both the sampling procedures and the biological processes of interest. For a study involving radio tagging as well as traditional tags, a realistic model might include parameters representing survival, emigration, radio failure, relocation and recapture rates, each of which could be time- and age-specific. On the other hand, models that include most or all of these parameter types may not be identifiable or may produce estimators with poor precision.

Radio failure was known to occur in the snail kite study, also it was suspected that relocation rates were lower for dead than living birds, because dead birds may have been submerged in water with nonfunctioning radios (Bennetts et al. 1999). The models investigated by Nasution et al. (2001) for the snail kite data therefore allowed for radio failure, seasonal variation in survival, and different telemetry relocation rates for live and dead birds. Both permanent and temporary emigration were assumed to be negligible, however. To derive a model for the black bear data, we follow the approach used by Nasution et al. (2001), though the parameter types are not the same. For the black bears, the parameters should account for temporary censoring and possibly permanent emigration (Sorensen and Powell 1998).

We use the parameterization of Tsai, Pollock, and Brownie (1999) to model permanent and temporary emigration. Permanent emigration is assumed to occur at the start of the period between telemetry surveys. Temporary censoring (temporary failure to relocate an 
animal) is assumed to result from random movement of bears in and out of the study area (or an area where topography prevents relocation). Because temporary censoring affects living but not dead animals, the result is lower relocation rates for living animals. Other reasons given by Tsai et al. (1999) for relocation rates to differ by mortality status are that radios may be destroyed or removed when animals are killed by predators or by hunters.

Tsai et al. (1999) demonstrated the potential for bias in telemetry estimates of survival when relocation rates differ by mortality status. Nevertheless, most analyses of telemetry data have assumed equality of relocation rates for living and dead animals (e.g., Pollock, Bunck, Winterstein, and Chen 1995; Sorensen and Powell 1998; Bennetts et al. 1999). We believe this is due in part to concerns about nonidentifiability and estimator precision if relocation rates are allowed to be different. As some of the interesting candidate models for the black bear data include different relocation rates as well as permanent emigration, nonidentifiability could cause problems in the model selection process. Checking identifiability is not easy, however, because of the complexity of the model structure and the number of parameter types. Even harder is checking estimability for a given dataset. Estimability is not addressed here; a numerical approach to determine the number of independently estimable parameters for a given dataset was described by Viallefont, Lebreton, Reboulet, and Gory (1998).

Catchpole, Freeman, and Morgan (1996); Catchpole and Morgan (1997, 2001); Catchpole, Morgan, and Freeman (1998); and Catchpole, Kgosi, and Morgan (2001) described methods for investigating identifiability of capture-recapture models. Catchpole and Morgan (1997) noted that overparameterization, or parameter redundancy, is the most obvious cause of nonidentifiability. For a general class of nonlinear models, they showed that overparameterization is equivalent to singularity of the information matrix of the corresponding likelihood, and provided an expression for the information matrix that is useful in checking singularity for product multinomial likelihoods. Models that are "near-singular" or close to being nonidentifiable were considered by Catchpole et al. (2001). They presented a counterintuitive case where reducing the number of parameters in an identified model leads to a nonidentifiable submodel. This example is important because it is usually assumed that parsimony (or reducing the number of parameters) will automatically lead to better identified models and so to estimators with increased precision. Their example also explains the poor performance of estimators for an identified "full" model that is close to a nonidentifiable "reduced" model because there is little variation across years in the survival parameter.

In this article, we describe models for the combined analysis of radio-telemetry and recapture data such as the black bear data. We focus on models that allow separate estimation of emigration and survival and use the methods of Catchpole and Morgan (1997, 2001) to investigate parameter redundancy of these models. We examine the effect of allowing different relocation rates for dead and living animals on model identifiability and estimator precision. The effect of allowing between- and within-year variation in survival is also studied. Results are applied to the analysis of the black bear data. 


\section{MODELS FOR THE COMBINED ANALYSIS}

Following Nasution et al. (2001), we present a likelihood for data from studies where all animals receive traditional tags and some also receive radio tags. Recapture surveys occur annually, followed by the release of tagged animals, and telemetry surveys occur each year at the same time as the recapture survey and at regular intervals during the year. Recapture and telemetry efforts are assumed to be independent and to extend to animals in a common area, so that emigration occurs when an animal leaves the study area. The black bear study covered an area of more than $400 \mathrm{~km}^{2}$ (Sorensen and Powell 1998). Average home range sizes are about $40 \mathrm{~km}^{2}$ and $20 \mathrm{~km}^{2}$ for male and female bears, respectively, and traps were distributed to maximize access to all animals in the population.

In order to minimize the occurrence of radio failure, bears were tracked to dens in order to change batteries or to replace radio collars (Sorensen and Powell 1998). We therefore assume that radio failure does not occur. Results for models that include radio failure were given by Nasution (2000). We assume that radio-tagged animals that die are relocated during the next telemetry survey or not at all. We also assume homogeneity of survival and recapture rates for all tagged animals, and of relocation probabilities for live animals, as well as for dead animals, at a given sampling occasion.

Under the conditions above, the joint likelihood for the telemetry and recapture data is obtained by modifying the likelihood of Nasution et al. (2001) to account for true survival and emigration. We let $K$ represent the number of annual recapture occasions and $k$ the number of telemetry surveys per year. More specifically, initial captures occur at the start of year 1 , followed by capture-recapture occasions at the start of years $2, \ldots, K+1$; also the first telemetry survey is at sampling time 2 in year 1 , and the last is at sampling time 1 in year $K+1$. For brevity, an animal with both a radio and traditional tag is referred to as a radioed animal, and one with only the traditional tag is a nonradioed animal.

The parameters in the model are:

$\phi_{i, j}=$ the probability that an animal alive after sample $j$ in year $i$, survives to the next sampling time $(i=1, \ldots, K, j=1, \ldots, k)$.

$F_{i, j}=$ the probability that an animal stays in the study area from sampling time $j$ in year $i$ to the next sampling time $(i=1, \ldots, K, j=1, \ldots, k)$.

$p_{l i, j}=$ the probability that a radioed animal that is alive in the study area at sampling time $j$ in year $i$ is relocated by radio.

$p_{d i, j}=$ the probability that a radioed animal that has recently died in the study area is relocated by radio in year $i$ at sampling time $j$.

$p_{s i}=$ the probability a tagged animal alive in the study area at year $i$ is recaptured at year $i(i=2, \ldots, K+1)$.

The likelihood for the combined datasets is

$$
L=L_{1} \times L_{2} \times L_{3}
$$

where each of the components $L_{1}, L_{2}, L_{3}$ is a product of independent multinomials. 
$L_{1}$ is the contribution from animals that receive only traditional tags.

$$
L_{1}=\prod_{i=1}^{K}\left\{\frac{R_{i}^{s} !}{\prod_{j=i+1}^{K+1} m_{i, j}^{s} !}\left[\prod_{j=i+1}^{K+1} \pi_{s i, j}^{m_{i, j}^{s}}\right] \chi_{i}^{s\left(R_{i}^{s}-r_{i}^{s}\right)}\right\}
$$

with

$R_{i}^{s}=$ the number of nonradioed animals released at the start of year $i$, including newly and previously tagged animals, $(i=1, \ldots, K)$.

$r_{i}^{s}=$ the total number of nonradioed animals ever recaptured of the $R_{i}^{s}$ animals released at the start of year $i(i=1, \ldots, K)$.

$m_{i, j}^{s}=$ the number of nonradioed animals released at the start of year $i$ that are next recaptured at the start of year $j(i=1, \ldots, K, j=i+1, \ldots, K+1)$.

$\pi_{s i, j}=$ the probability a nonradioed animal alive at year $i$ is next recaptured at the start of year $j, i=1, \ldots, K, j=i+1, \ldots, K+1$.

$\chi_{i}^{s}=$ the probability a nonradioed animal released at the start of year $i$ is never recaptured again $(i=1, \ldots, K)$, defined recursively by $1-\chi_{i}^{s}=\pi_{s i, i+1}+$ $\pi_{s i, i+1} \frac{\left(1-p_{s, i+1}\right)}{p_{s, i+1}}\left(1-\chi_{i+1}^{s}\right), \chi_{K+1}^{s}=1$.

$L_{2}$ is the contribution from animals with both radios and traditional tags.

$$
L_{2}=\prod_{i=1}^{K} \prod_{j=1}^{k}\left\{\frac{R_{i, j}^{r} !}{\prod_{i^{\prime}, j^{\prime}}\left[m_{i, j, i^{\prime}, j^{\prime}}^{r} ! d_{i, j, i^{\prime}, j^{\prime}} !\right]}\left[\prod_{i^{\prime}, j^{\prime}} \pi_{l i, j, i^{\prime}, j^{\prime}}^{m_{i, j, j^{\prime}}^{r}} \pi_{d i, j, i^{\prime}, j^{\prime}}^{d_{i, j, i^{\prime}, j^{\prime}}}\right] \chi_{i, j}^{r_{i, j}^{\left(R^{r}-r_{i, j}^{r}\right)}}\right\}
$$

with

$R_{i, j}^{r}=$ the number of radioed animals released after the $j$ th sample in year $i$ including newly and previously tagged animals $(i=1, \ldots, K, j=1, \ldots, k)$.

$r_{i, j}^{r}=$ the total number of radioed animals subsequently relocated, alive or dead, of the $R_{i, j}^{r}$ animals released after the $j$ th sample in year $i(i=1, \ldots, K$, $j=1, \ldots, k)$.

$m_{i, j, i^{\prime}, j^{\prime}}^{r}=$ the number of radioed animals relocated by radio, or newly released, at the $j$ th sample in year $i$ and next relocated alive in the $j^{\prime}$ th sample in year $i^{\prime}$.

$d_{i, j, i^{\prime}, j^{\prime}}=$ the number of radioed animals relocated by radio, or newly released, at the $j$ th sample in year $i$, and next relocated dead in the $j^{\prime}$ th sample in year $i^{\prime}$.

$\pi_{l i, j, i^{\prime}, j^{\prime}}=$ the probability that a radioed animal last relocated at sample $j$ in year $i$, is next relocated alive at sample $j^{\prime}$ in year $i^{\prime} ; i^{\prime}=i, \ldots, K+1$, and $j^{\prime}=$ $j+1, \ldots, k$ if $i^{\prime}=i$ or $j^{\prime}=1, \ldots, k$ if $i^{\prime}>i$ or $j^{\prime}=1$ if $i^{\prime}=K+1$.

$\pi_{d i, j, i^{\prime}, j^{\prime}}=$ the probability that a radioed animal last relocated at sample $j$ in year $i$ is next relocated dead at sample $j^{\prime}$ in year $i^{\prime} ; i^{\prime}=i, \ldots, K$, and $j^{\prime}=j+1, \ldots, k$ if $i^{\prime}=i$ or $j^{\prime}=1, \ldots, k$ if $i^{\prime}>i$ or $j^{\prime}=1$ if $i^{\prime}=K+1$.

$\chi_{i, j}^{r}=$ the probability a radioed animal released at sample $j$ in year $i$ is never relocated again by radio and never recaptured $(i=1, \ldots, K, j=1, \ldots, k)$, defined by $1-\chi_{i, j}^{r}=F_{i, j}\left[\left(1-S_{i, j}\right) p_{d i, j+1}+S_{i, j} p_{l i, j+1}+S_{i, j}\left(1-p_{l i, j+1}\right)(1-\right.$ $\left.\left.\chi_{i, j+1}^{r}\right)\right], \chi_{K+1,1}^{r}=1$. 
Under models where radio failure does occur, $L_{2}$ contains additional terms to account for radioed animals that are recaptured with a nonfunctioning radio (Nasution 2000). The events "not relocated but recaptured with a functioning radio" could also be included in $L_{2}$ (Nasution 2000).

The third component of the likelihood is the contribution from radioed animals that are recaptured as well as relocated at the start of the year.

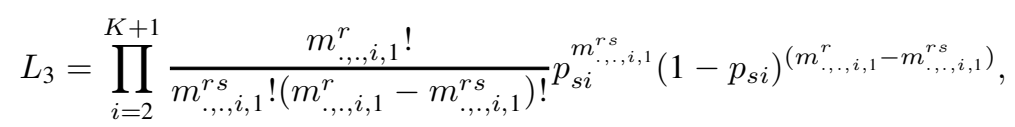

Table 1. Selected Cell Probabilities for a Study With Two Annual Recapture Samples and Six-Monthly Telemetry Surveys

\begin{tabular}{|c|c|c|c|}
\hline \multirow[b]{2}{*}{ Parameter } & \multicolumn{2}{|c|}{ When } & \multirow[b]{2}{*}{ Cell probability } \\
\hline & Last seen & Next seen & \\
\hline$\pi_{/ 1,1,1,2}$ & 1,1 & 1,2 & $\phi_{1,1} F_{1,1} p_{/ 1,2}$ \\
\hline$\pi_{/ 1,1,2,1}$ & 1,1 & 2,1 & $\phi_{1,1} F_{1,1} q_{/ 1,2} \phi_{1,2} F_{1,2} p_{/ 2,1}$ \\
\hline \multirow[t]{2}{*}{$\pi_{/ 1,1,2,2}$} & 1,1 & 2,2 & $\phi_{1,1} F_{1,1} q_{/ 1,2}$ \\
\hline & & & $\phi_{1,2} F_{1,2} q_{/ 2,1} \phi_{2,1} F_{2,1} p_{l 2,2}$ \\
\hline \multirow[t]{2}{*}{$\pi_{/ 1,1,3,1}$} & 1,1 & 3,1 & $\phi_{1,1} F_{1,1} q_{11,2} \phi_{1,2} F_{1,2} q_{/ 2,1}$ \\
\hline & & & $\phi_{2,1} F_{2,1} q_{/ 2,2} \phi_{2,2} F_{2,2} p_{/ 3,1}$ \\
\hline$\pi_{/ 1,2,2,1}$ & 1,2 & 2,1 & $\phi_{1,2} F_{1,2} p_{/ 2,1}$ \\
\hline$\pi_{/ 1,2,2,2}$ & 1,2 & 2,2 & $\phi_{1,2} F_{1,2} q_{/ 2,1} \phi_{2,1} F_{2,1} p_{/ 2,2}$ \\
\hline \multirow[t]{2}{*}{$\pi_{/ 1,2,3,1}$} & 1,2 & 3,1 & $\phi_{1,2} F_{1,2} q_{/ 2,1}$ \\
\hline & & & $\phi_{2,1} F_{2,1} q_{/ 2,2} \phi_{2,2} F_{2,2} p_{l 3,1}$ \\
\hline$\pi_{/ 2,1,2,2}$ & 2,1 & 2,2 & $\phi_{2,1} F_{2,1} p_{I 2,2}$ \\
\hline$\pi_{/ 2,1,3,1}$ & 2,1 & 3,1 & $\phi_{2,1} F_{2,1} q_{/ 2,2} \phi_{2,2} F_{2,2} p_{/ 3,1}$ \\
\hline$\pi_{/ 2,2,3,1}$ & 2,2 & 3,1 & $\phi_{2,2} F_{2,2} p_{/ 3,1}$ \\
\hline$\pi_{d 1,1,1,2}$ & 1,1 & 1,2 & $\left(1-\phi_{1,1}\right) F_{1,1} p_{d 1,2}$ \\
\hline$\pi_{d 1,1,2,1}$ & 1,1 & 2,1 & $\phi_{1,1} F_{1,1} q_{/ 1,2}\left(1-\phi_{1,2}\right) F_{1,2} p_{d 2,1}$ \\
\hline$\pi_{d 1,1,2,2}$ & 1,1 & 2,2 & $\phi_{1,1} F_{1,1} q_{l 1,2}$ \\
\hline$\pi_{d 1,1,3,1}$ & 1,1 & 3,1 & $\begin{array}{l}\phi_{1,2} F_{1,2} q_{l 2,1}\left(1-\phi_{2,1}\right) F_{2,1} p_{d 2,2} \\
\phi_{1,1} F_{1,1} q_{l 1,2} \phi_{1,2} F_{1,2} q_{l 2,1} \\
\phi_{2,1} F_{2,1} q_{l 2,2}\left(1-\phi_{2,2}\right) F_{2,2} p_{d 3,1}\end{array}$ \\
\hline$\pi_{d 1,2,2,1}$ & 1,2 & 2,1 & $\left(1-\phi_{1,2}\right) F_{1,2} p_{d 2,1}$ \\
\hline$\pi_{d 1,2,2,2}$ & 1,2 & 2,2 & $\phi_{1,2} F_{1,2} q_{/ 2,1}\left(1-\phi_{2,1}\right) F_{2,1} p_{d 2,2}$ \\
\hline \multirow[t]{2}{*}{$\pi_{d 1,2,3,1}$} & 1,2 & 3,1 & $\phi_{1,2} F_{1,2} q_{/ 2,1}$ \\
\hline & & & $\phi_{2,1} F_{2,1} q_{/ 2,2}\left(1-\phi_{2,2}\right) F_{2,2} p_{d 3,1}$ \\
\hline$\pi_{d 2,1,2,2}$ & 2,1 & 2,2 & $\left(1-\phi_{2,1}\right) F_{2,1} p_{d 2,2}$ \\
\hline$\pi_{d 2,1,3,1}$ & 2,1 & 3,1 & $\phi_{2,1} F_{2,1} q_{/ 2,2}\left(1-\phi_{2,2}\right) F_{2,2} p_{d 3,1}$ \\
\hline$\pi_{d 2,2,3,1}$ & 2,2 & 3,1 & $\left(1-\phi_{2,2}\right) F_{2,2} p_{d 3,1}$ \\
\hline$\pi_{s 1,2}$ & 1 & 2 & $\phi_{1,1} \phi_{1,2} F_{1,1} F_{1,2} p_{s 2}$ \\
\hline$\pi_{s 1,3}$ & 1 & 3 & $\phi_{1,1} \phi_{1,2} F_{1,1} F_{1,2} q_{s 2} \phi_{2,1} \phi_{2,2} F_{2,1} F_{2,2} p_{s 3}$ \\
\hline$\pi_{s 2,3}$ & 2 & 3 & $\phi_{2,1} \phi_{2,2} F_{2,1} F_{2,2} p_{s 3}$ \\
\hline
\end{tabular}


where

$m_{., ., i, 1}^{r}=$ the total number of radioed animals relocated by radio at the start of year $i$

$m_{., ., i, 1}^{r s}=$ the total number of the $m_{., ., i, 1}^{r}$ radioed animals relocated by radio at the start of year $i$ that are also recaptured at the start of year $i(i=2, \ldots, K+1)$.

The structure of the multinomial cell probabilities $\pi_{l i, j, i^{\prime}, j^{\prime}}$ and $\pi_{d i, j, i^{\prime}, j^{\prime}}$ in component $L_{2}$, and $\pi_{s i, j}$ in component $L_{1}$, is indicated in Table 1 for a study with release of tagged animals followed by $K=2$ annual recapture samples and $k=2$ telemetry surveys per year. Each of the parameters $\phi, F, p_{l}, p_{d}$, and $p_{s}$ in Table 1 is assumed to be time specific.

\section{PARAMETER IDENTIFICATION}

Catchpole and Morgan $(1997,2001)$ presented the general form of the expected information matrix for a multinomial distribution $\left(n, \pi_{1}, \ldots, \pi_{m}\right)$, where $\pi_{1}, \ldots, \pi_{m}$ are functions of $\boldsymbol{\theta}=\left(\theta_{1}, \ldots, \theta_{l}\right)^{T}$, as

$$
\mathcal{I}=\mathbf{D} \Pi^{-1} \mathbf{D}^{T}
$$

where $\Pi=\operatorname{diag}(\boldsymbol{\mu})$ such that $\mu_{j}=n \pi_{j}$ and $\frac{\partial \mu_{j}}{\partial \theta_{i}}=$ the $(i, j)$ th element of $\mathbf{D}$. This general form for $\mathcal{I}$ can be extended in an obvious way to product multinomial distributions such as the likelihood $L$ in Equation (2.1). To show identifiability of a model obtained from $L$ in (2.1) by imposing specific constraints on the parameters, we must show that the corresponding information matrix $\mathcal{I}$ is nonsingular (Catchpole and Morgan 1997), or equivalently, that $\mathbf{D}$ is full row rank.

Determining singularity of $\mathcal{I}$ is simplified by noting that $L$ factors into three components, where the first component $L_{1}$ represents the outcomes for nonradioed animals, and the components $L_{2} \times L_{3}$ relate to outcomes for radioed animals. Accordingly, we partition $\mathbf{D}$ in Equation (3.1) so that the first partition $\mathbf{D}^{s}$ contains the elements resulting from $L_{1}$, and the second partition $\mathbf{D}^{r}$ contains elements resulting from $L_{2}$ and $L_{3}$. Thus,

$$
\begin{aligned}
\mathcal{I}=\mathbf{D \Pi}^{-1} \mathbf{D}_{a \times a}^{T}= & {\left[\begin{array}{lll}
\mathbf{D}_{a \times n_{s}}^{s} & \mid & \mathbf{D}^{r}{ }_{a \times n_{r}}
\end{array}\right] } \\
& \times\left[\begin{array}{cc}
\boldsymbol{\mu}^{s} & \mathbf{0} \\
\mathbf{0} & \boldsymbol{\mu}^{r}
\end{array}\right]^{-1}\left[\begin{array}{lll}
\mathbf{D}_{a \times n_{s}}^{s} & \mathbf{D}_{a \times n_{r}}^{r}
\end{array}\right]^{T}
\end{aligned}
$$

with $a=$ the number of distinct parameters in $L, n_{s}=$ the total number of possible outcomes for nonradioed animals, and $n_{r}=$ the total number of possible outcomes for radioed animals.

Defining $\mathcal{I}_{s}=\mathbf{D}^{s} \boldsymbol{\mu}^{s} \mathbf{D}^{s T}$ and $\mathcal{I}_{r}=\mathbf{D}^{r} \boldsymbol{\mu}^{r} \mathbf{D}^{r T}$, and combining Equations (3.1) and (3.2), leads to (Azzalini 1996, p. 73):

$$
\mathcal{I}=\mathcal{I}_{s}+\mathcal{I}_{r}
$$

The cohorts of nonradioed and radioed animals are independent and hence the two matrices $\mathbf{D}^{r}$ and $\mathbf{D}^{s}$ relate to mutually exclusive outcomes. Also, $\mathbf{D}^{r}$ is a function of $\boldsymbol{\theta}^{r}$, and $\mathbf{D}^{s}$ is 


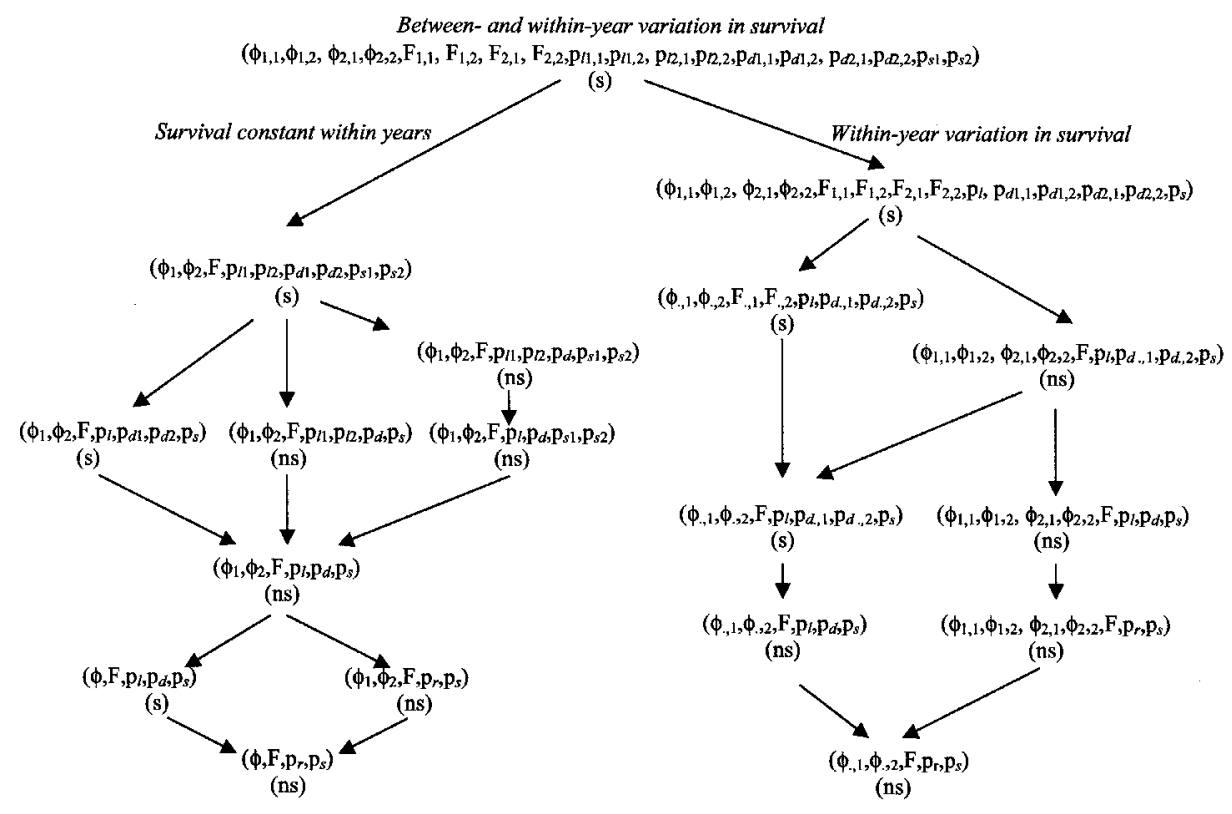

Figure 1. Condition of the information matrix for two sets of models: $(n s)$ and $(s)$ indicate a nonsingular and a singular matrix, respectively. The first subscript on a parameter indicates between-year dependence and the second subscript indicates within-year dependence.

a function of $\boldsymbol{\theta}^{s}$, where each element of $\boldsymbol{\theta}^{s}$ is also an element of $\boldsymbol{\theta}^{r}$. However, $\boldsymbol{\theta}^{r}$ contains at least one element (specifically, a relocation rate) which is not an element of $\boldsymbol{\theta}^{s}$. D will therefore have full row rank, $r(\mathbf{D})=a$, if $\mathbf{D}^{r}$ is full row rank. The argument is simple. If $\mathbf{D}_{\left(a \times n_{r}\right)}^{r}$ is full row rank, then there is at least one nonsingular $a \times a$ square matrix which is a subsidiary of $\mathbf{D}^{r}$ (Searle 1982, p. 171), and which must also be a subsidiary of $\mathbf{D}$.

If row rank $\mathbf{D}^{r}$ is deficient, we can consider further partitions of matrices $\mathbf{D}^{s}$ and $\mathbf{D}^{r}$. Let $\left[\mathbf{D}^{s}\right]^{T}=\left[\left[\mathbf{D}_{\star}^{s}\right]^{T} \mid \mathbf{0}^{T}\right]$ and $\left[\mathbf{D}^{r}\right]^{T}=\left[\left[\mathbf{D}_{1}^{r}\right]^{T} \mid\left[\mathbf{D}_{2}^{r}\right]^{T}\right]$ such that both $\mathbf{D}_{\star}^{s}$ and $\mathbf{D}_{1}^{r}$ are functions of $\boldsymbol{\theta}^{s}$ and $\mathbf{D}_{2}^{r}$ is a function of the elements in $\boldsymbol{\theta}$ that are contained in $\boldsymbol{\theta}^{r}$ but not in $\boldsymbol{\theta}^{s}$. As long as both $\mathbf{D}_{\star}^{s}$ and $\mathbf{D}_{2}^{r}$ are full row rank matrices, matrix $\mathbf{D}$ will have full row rank (by Theorem 6 of Catchpole and Morgan 1997). Therefore there are two conditions that will guarantee the full row rank of $\mathbf{D}$ and consequently the full rank of the expected information matrix $\mathcal{I}$ : (1) full row rank of $\mathbf{D}^{r}$, equivalently, full rank of $\mathcal{I}_{r}$, and (2) full row rank of both $\mathbf{D}_{\star}^{s}$ and $\mathbf{D}_{2}^{r}$. If at least one of these conditions is satisfied, matrix $\mathbf{D}$ will have full row rank and equivalently $\mathcal{I}$ will be nonsingular.

We examine parameter redundancy for two sets of models. Comparisons among the first set (shown on the left of Figure 1) focus on between-year variation in survival, relocation, and recapture rates. Comparisons among the second set (to the right of Figure 1) emphasize the effect of within-year variation, which is of interest in the black bear data. As in Table 1, we assume $K=2$ and $k=2$. Algebraic formulas are simpler in this situation and results concerning identifiability will carry over to models for studies with $K, k>2$.

The models examined are represented by a list of parameters in parentheses (see Figure 
1). Two subscripts on a parameter denote between- and within-year variation (e.g., $\phi_{i, j}$ ), a period (.) as the first subscript indicates within-year variation only (e.g., $\phi ., j$ ), a single subscript denotes between-year variation only (e.g., $\phi_{i}$ ), and no subscript indicates that the parameter is constant both within and between years (e.g., $\phi$ ). Models with different relocation rates for live and dead animals are represented by including both $p_{l}$ and $p_{d}$ in the parameter list, while models with relocation rate independent of mortality status are indicated using a single parameter $p_{r}$ in the list.

Entries of the derivative matrix $\mathbf{D}$ were obtained for each of the models. The rank of $\mathbf{D}$, and hence singularity of $\mathcal{I}$, was determined analytically for each. Results in Figure 1 show that the general model with all parameters time specific is singular, as are some of the reduced parameter models. All of the singular models include different relocation rates for live and dead animals. We see that when $F$ is constant and the nature of the time dependency is the same for survival and $p_{d}$, the model contains redundant parameters. For example, the five-parameter model with $\phi$ and $p_{d}$ both constant across time is singular. Also, when $\phi$ and $p_{d}$ vary within years, but not between years, as in model $\left(\phi_{., 1}, \phi_{., 2}, F, p_{l}, p_{d ., 1}, p_{d ., 2}, p_{s}\right)$, the information matrix is singular. From the cell probabilities in Table 1, we see that if $F, \phi$, and $p_{d}$, are all constant across time, then these parameters always occur either as the product $(1-\phi) F p_{d}$, or as $\phi F$, leading to nonidentifiability of the model. (Analogous results hold for models which allow an additional parameter for radio failure; Nasution 2000).

Several of the models in Figure 1 display the phenomenon reported by Catchpole et al. (2001), where reducing the number of parameters in a nonsingular model results in one that is singular. Thus, the six-parameter model $\left(\phi_{1}, \phi_{2}, F, p_{l}, p_{d}, p_{s}\right)$ with year-specific survival is nonsingular, but the reduced model with constant survival $\left(\phi, F, p_{l}, p_{d}, p_{s}\right)$ is singular. In the terminology of Catchpole et al. (2001), model $\left(\phi_{1}, \phi_{2}, F, p_{l}, p_{d}, p_{s}\right)$ is near-singular and we expect estimators under this model to have poor precision when the true survival rates are similar in magnitude. We investigated properties of the model $\left(\phi_{1}, \phi_{2}, F, p_{l}, p_{d}, p_{s}\right)$, for several sets of parameter values, by determining the smallest eigenvalue of $\mathcal{I}(\boldsymbol{\theta})$, and by obtaining large-sample estimator variances and covariances from the elements of $\mathbf{V}(\boldsymbol{\theta})=$ $\mathcal{I}(\boldsymbol{\theta})^{-1}$. For comparison, we also evaluated the properties of the model $\left(\phi_{1}, \phi_{2}, F, p_{r}, p_{s}\right)$ which assumes equal relocation rates for live and dead animals. Parameter values used in the calculations are $\phi_{1}=0.6, F=0.95$, and $p_{l}=0.9$ for model $\left(\phi_{1}, \phi_{2}, F, p_{l}, p_{d}, p_{s}\right)$ or $p_{r}=0.9$ for model $\left(\phi_{1}, \phi_{2}, F, p_{r}, p_{s}\right)$. In addition, we take $\phi_{2}=0.5,0.6$, and $0.7, p_{d}=0.8$ and 0.9 , and $p_{s}=0.2,0.6$, and 0.8. The numbers of animals released are $R_{1,1}^{r}=100$, $R_{1,2}^{r}=100, R_{2,1}^{r}=100, R_{2,2}^{r}=100, R_{1}^{s}=1,000$, and $R_{2}^{s}=1,000$. These numbers are larger than is usually feasible, but are large enough to ensure that variance calculations based on $\mathcal{I}(\boldsymbol{\theta})^{-1}$ are accurate.

Elements of $\mathcal{I}(\boldsymbol{\theta})$ were obtained for each set of parameter values using Equation (3.1). Eigenvalues of $\mathcal{I}(\boldsymbol{\theta})$, and $\mathbf{V}(\boldsymbol{\theta})=\mathcal{I}(\boldsymbol{\theta})^{-1}$ were obtained using routines in the FORTRANIMSL library (IMSL, Inc. 1987). Large-sample variances and correlations, for example, $V\left(\hat{\phi}_{1}\right), V\left(\hat{\phi}_{2}\right)$, and $r\left(\hat{\phi}_{1}, \hat{\phi}_{2}\right)=\frac{\operatorname{cov}\left(\hat{\phi}_{1}, \hat{\phi}_{2}\right)}{\sqrt{V\left(\hat{\phi}_{1}\right)} \sqrt{V\left(\hat{\phi}_{2}\right)}}$, were then obtained using the appropriate diagonal and off-diagonal elements of $\mathbf{V}(\boldsymbol{\theta})$. The smallest eigenvalue of $\mathcal{I}(\boldsymbol{\theta})$, and selected variances and correlations, for the different sets of parameter values, are presented in Tables 
Table 2. Values of the Smallest Eigenvalue of $\mathcal{I}(\boldsymbol{\theta})$, Asymptotic Variances and Correlations, for the Model $\left(\phi_{1}, \phi_{2}, F, p_{l}, p_{d}, p_{s}\right)$

\begin{tabular}{cccccccccc}
\hline \hline$\phi_{2}$ & $p_{d}$ & $p_{s}$ & $\min (\lambda)$ & $V\left(\hat{\phi}_{1}\right)$ & $V\left(\hat{\phi}_{2}\right)$ & $r\left(\hat{\phi}_{1}, \hat{\phi}_{2}\right)$ & $r\left(\hat{\phi}_{1}, \hat{F}\right)$ & $r\left(\hat{\phi}_{1}, \hat{p}_{d}\right)$ & $r\left(\hat{F}, \hat{p}_{d}\right)$ \\
\hline 0.5 & 0.8 & 0.2 & 4.1 & 0.0225 & 0.0181 & 0.940 & -0.966 & 0.962 & -0.992 \\
& & 0.6 & 4.2 & 0.0217 & 0.0162 & 0.973 & -0.986 & 0.980 & -0.992 \\
& & 0.8 & 4.2 & 0.0215 & 0.0157 & 0.981 & -0.991 & 0.984 & -0.992 \\
& 0.9 & 0.2 & 5.5 & 0.0146 & 0.0124 & 0.912 & -0.948 & 0.946 & -0.992 \\
& & 0.6 & 5.7 & 0.0139 & 0.0106 & 0.958 & -0.978 & 0.973 & -0.992 \\
& & 0.8 & 5.7 & 0.0137 & 0.0102 & 0.970 & -0.986 & 0.980 & -0.992 \\
0.6 & 0.8 & 0.2 & 0.0 & & & & & & \\
& & 0.6 & 0.0 & & & & & & \\
& & 0.8 & 0.0 & & & & & & \\
& & & & & & & & & \\
& 0.9 & 0.2 & 0.0 & & & & & & \\
& 0.6 & 0.0 & & & & & & \\
& 0.8 & 0.0 & & & & & & \\
0.7 & 0.8 & 0.2 & 4.7 & 0.0152 & 0.0167 & 0.921 & -0.954 & 0.949 & -0.988 \\
& 0.6 & 4.9 & 0.0134 & 0.0166 & 0.964 & -0.981 & 0.972 & -0.988 \\
& 0.8 & 5.0 & 0.0130 & 0.0166 & 0.976 & -0.987 & 0.977 & -0.988 \\
& & & & & & & & & \\
& 0.9 & 0.2 & 5.7 & 0.0112 & 0.0114 & 0.892 & -0.938 & 0.936 & -0.989 \\
& 0.6 & 6.1 & 0.0095 & 0.0114 & 0.949 & -0.973 & 0.966 & -0.989 \\
& 0.8 & 6.2 & 0.0091 & 0.0113 & 0.965 & -0.982 & 0.973 & -0.989 \\
\hline
\end{tabular}

2 and 3 , for models $\left(\phi_{1}, \phi_{2}, F, p_{l}, p_{d}, p_{s}\right)$ and $\left(\phi_{1}, \phi_{2}, F, p_{r}, p_{s}\right)$, respectively.

For model $\left(\phi_{1}, \phi_{2}, F, p_{l}, p_{d}, p_{s}\right)$, when $\phi_{1}=\phi_{2}=0.6$ the smallest eigenvalue of $\mathcal{I}(\boldsymbol{\theta})$ is 0 (Table 2), which agrees with the result in Figure 1 that the information matrix $\mathcal{I}$ is singular for the model $\left(\phi, F, p_{l}, p_{d}, p_{s}\right)$. For $\phi_{2}=0.5,0.7$, the smallest eigenvalue is close to 0 and correlations between estimators of $\phi_{1}, \phi_{2}, F$ and $p_{d}$ are strong. For this nearsingular model, estimators are poorly behaved for all of the parameter values investigated. In contrast, model $\left(\phi_{1}, \phi_{2}, F, p_{r}, p_{s}\right)$ shows no evidence of parameter redundancy (Table $3)$. The smallest eigenvalue of $\mathcal{I}$ is two orders of magnitude greater for the model with $p_{l}$ and $p_{d}$ assumed equal compared to the model in which $p_{l}$ and $p_{d}$ are estimated separately. Also, when $p_{l}$ and $p_{d}$ are assumed equal, correlations among estimators are closer to 0 , and $V\left(\hat{\phi}_{1}\right), V\left(\hat{\phi}_{2}\right)$ are smaller than values in Table 2 indicating improved precision for estimating survival. The practical implications are that every effort should be made to relocate dead as well as living animals, because models that permit different relocation rates are ill-conditioned. Consistent and high effort across years, so that $p_{d}$ and $p_{l}$ are not only similar but also constant across time, will justify the use of parsimonious models with good properties.

The third model investigated, model $\left(\phi_{1,1}, \phi_{1,2}, \phi_{2,1}, \phi_{2,2}, F, p_{l}, p_{d}, p_{s}\right)$, is also nearsingular (Table 4). If the values of the survival rates are similar within and between years, the precision of estimators will be poor under this model. The parameter values used in Table 4 represent a strong seasonal effect, however, and so the models are not as close to singular as those represented in Table 2 . The smallest eigenvalues are therefore intermediate 
Table 3. Values of the Smallest Eigenvalue of $\mathcal{I}(\boldsymbol{\theta})$, Asymptotic Variances and Correlations, for the Model $\left(\phi_{1}, \phi_{2}, F, p_{r}, p_{s}\right)$

\begin{tabular}{ccccccccc}
\hline \hline$\phi_{2}$ & $p_{s}$ & $\min (\lambda)$ & $V\left(\hat{\phi}_{1}\right)$ & $V\left(\hat{\phi}_{2}\right)$ & $r\left(\hat{\phi}_{1}, \hat{\phi}_{2}\right)$ & $r\left(\hat{\phi}_{1}, \hat{F}\right)$ & $r\left(\hat{\phi}_{1}, \hat{p}_{r}\right)$ & $r\left(\hat{F}, \hat{p}_{r}\right)$ \\
\hline 0.5 & 0.2 & 505.7 & 0.0016 & 0.0013 & 0.189 & -0.281 & 0.174 & -0.519 \\
& 0.6 & 654.6 & 0.0008 & 0.0010 & 0.326 & -0.507 & 0.278 & -0.516 \\
& 0.8 & 717.9 & 0.0006 & 0.0003 & 0.382 & -0.625 & 0.333 & -0.515 \\
0.6 & 0.2 & 511.8 & 0.0015 & 0.0015 & 0.178 & -0.281 & 0.170 & -0.501 \\
& 0.6 & 667.4 & 0.0007 & 0.0007 & 0.326 & -0.516 & 0.273 & -0.497 \\
& 0.8 & 729.6 & 0.0006 & 0.0006 & 0.394 & -0.628 & 0.323 & -0.496 \\
0.7 & 0.2 & 536.7 & 0.0014 & 0.0015 & 0.162 & -0.280 & 0.164 & -0.483 \\
& 0.6 & 692.5 & 0.0007 & 0.0009 & 0.317 & -0.521 & 0.264 & -0.476 \\
& 0.8 & 752.8 & 0.0005 & 0.0007 & 0.398 & -0.625 & 0.308 & -0.474 \\
\hline \multicolumn{1}{c}{$\phi_{1}=0.6, F=0.95, p_{r}=0.9$} & & & & &
\end{tabular}

between those in Tables 2 and 3 , and correlations between $\hat{p}_{d}$ and other estimators are stronger than for the model with a single relocation parameter $p_{r}$.

Using methods of Catchpole et al. (2001), we can obtain additional information about which parameters are poorly estimated in the near-singular model $\left(\phi_{1}, \phi_{2}, F, p_{l}, p_{d}, p_{s}\right)$ by determining the coefficients of parameters in the eigenvector corresponding to the smallest eigenvalue of $\mathcal{I}(\boldsymbol{\theta})$. As an example, we calculate the elements of this eigenvector for the model with parameter values $\phi_{1}=0.6, \phi_{2}=0.5, F=0.95, p_{l}=0.9, p_{d}=0.6$ and $p_{s}=0.6$, that is, the model represented in the second row of Table 2. The elements of this eigenvector are $(0.298,0.256,-0.475,-0.005,0.787,-0.002)$, corresponding to the parameters $\left(\phi_{1}, \phi_{2}, F, p_{l}, p_{d}, p_{s}\right)$. We see that $p_{l}$ and $p_{s}$ have small contributions to the eigenvector which indicates good precision of the corresponding estimators. In contrast, $p_{d}$ has the largest contribution to the eigenvector, followed by $F, \phi_{1}$ and $\phi_{2}$, indicating that these parameters are poorly estimated, and that there is some confounding between $p_{d}$ and the parameters $\phi_{1}, \phi_{2}$, and $F$.

\section{TELEMETRY AND TRAPPING STUDY ON BLACK BEARS}

We analyze data from the years 1981-1987 of the black bear study (Sorensen and Powell 1998). Between 1981 and 1986 a total of 62 bears were captured and marked with ear tags, and 46 of the 62 also received radio tags, though not always on the first capture occasion. Bears were trapped mainly in May and June. During the months when bears were active, efforts were made to relocate each bear every week. In December to April, telemetry surveys were infrequent. Sorensen and Powell (1998) showed that mortality during summer and fall was higher than in winter months, but obtained similar estimates of annual survival (58\% and 59\%) using monthly and annual summaries, respectively, of the telemetry records. To reflect seasonal variation, we use a biannual summary of the telemetry data, based on surveys in May-June and in November (about the time when the animals become inactive).

The numbers of tagged bears, recaptures, and relocations are limited and so fitted models must involve a small number of parameters. Parameters were therefore treated as 
Table 4. Values of the smallesteigenvalue of $\mathcal{I}(\boldsymbol{\theta})$, Asymptotic Variances and Correlations, for the Model $\left(\phi_{1,1}, \phi_{1,2}, \phi_{2,1}, \phi_{2,2}, F, p_{l}, p_{d}, p_{s}\right)$

\begin{tabular}{cccccccccc}
\hline \hline$\phi_{2}$ & $p_{d}$ & $p_{s}$ & $\min (\lambda)$ & $V\left(\hat{\phi}_{1,1}\right)$ & $V\left(\hat{\phi}_{1,2}\right)$ & $r\left(\hat{\phi}_{1,1}, \hat{\phi}_{1,2}\right)$ & $r\left(\hat{\phi}_{1,1}, \hat{F}\right)$ & $r\left(\hat{\phi}_{1,1}, \hat{p}_{d}\right)$ & $r\left(\hat{F}, \hat{p}_{d}\right)$ \\
\hline 0.5 & 0.8 & 0.2 & 45.9 & 0.00261 & 0.00157 & 0.209 & -0.585 & 0.555 & -0.913 \\
& & 0.6 & 46.0 & 0.00204 & 0.00150 & 0.131 & -0.712 & 0.655 & -0.914 \\
& & 0.8 & 46.0 & 0.00190 & 0.00149 & 0.109 & -0.752 & 0.687 & -0.915 \\
& 0.9 & 0.2 & 59.6 & 0.00234 & 0.00128 & 0.108 & -0.526 & 0.510 & -0.919 \\
& 0.6 & 59.6 & 0.00176 & 0.00121 & -0.007 & -0.664 & 0.619 & -0.921 \\
& 0.8 & 59.7 & 0.00162 & 0.00119 & -0.043 & -0.709 & 0.654 & -0.921 \\
0.6 & 0.8 & 0.2 & 36.9 & 0.00262 & 0.00164 & 0.234 & -0.606 & 0.579 & -0.923 \\
& 0.6 & 36.9 & 0.00204 & 0.00157 & 0.158 & -0.727 & 0.675 & -0.924 \\
& 0.8 & 36.9 & 0.00191 & 0.00156 & 0.139 & -0.761 & 0.702 & -0.905 \\
& & & & & & & & & \\
& 0.9 & 0.2 & 45.5 & 0.00239 & 0.00134 & 0.141 & -0.560 & 0.546 & -0.930 \\
& 0.6 & 45.5 & 0.00180 & 0.00126 & 0.031 & -0.690 & 0.648 & -0.931 \\
& 0.8 & 45.5 & 0.00167 & 0.00125 & 0.002 & -0.729 & 0.679 & -0.932 \\
& & & & & & & & & \\
0.7 & 0.8 & 0.2 & 33.7 & 0.00249 & 0.00164 & 0.218 & -0.596 & 0.569 & -0.921 \\
& 0.6 & 34.1 & 0.00186 & 0.00155 & 0.122 & -0.710 & 0.657 & -0.922 \\
& 0.8 & 34.2 & 0.00174 & 0.00153 & 0.100 & -0.741 & 0.680 & -0.922 \\
& & & & & & & & \\
& 0.9 & 0.2 & 40.9 & 0.00230 & 0.00134 & 0.132 & -0.557 & 0.544 & -0.928 \\
& 0.6 & 41.3 & 0.00167 & 0.00126 & 0.003 & -0.678 & 0.635 & -0.929 \\
& 0.8 & 41.4 & 0.00155 & 0.00124 & -0.029 & -0.712 & 0.660 & -0.929 \\
\hline
\end{tabular}

$\phi_{1}=\phi_{1,1} \phi_{1,2}=0.6, F=0.95, p_{l}=0.9$

For $\phi_{2}=0.5, \phi_{2,1}=0.5946$, and $\phi_{2,2}=0.8409$

For $\phi_{2}=0.6, \phi_{2,1}=0.6817$, and $\phi_{2,2}=0.8801$

For $\phi_{2}=0.7, \phi_{2,1}=0.7653$, and $\phi_{2,2}=0.9147$

constant across years as by Sorensen and Powell (1998), and fitted models focused on estimating seasonal survival while allowing different relocation rates according to mortality status, as well as season-specific emigration. Season-specific parameters are $\phi_{m}$ and $\phi_{n}$, the survival rates from May to November and from November to May, respectively; and $F_{m}$ and $F_{n}$, the fidelity rates in May and November, respectively. From models $\left(\phi_{., 1}, \phi_{., 2}, F_{., 1}, F_{., 2}, p_{l}, p_{d ., 1}, p_{d ., 2}, p_{s}\right)$ and $\left(\phi_{., 1}, \phi_{., 2}, F, p_{l}, p_{d ., 1}, p_{d ., 2}, p_{s}\right)$ in Figure 1 , we know that allowing $p_{d}$ to be season-specific will lead to non-identifiability, and so the relocation rates $p_{l}, p_{d}$, and the recapture rate $p_{s}$ were each assumed to be time independent.

Six different models, corresponding to different sets of constraints on the seven parameters, were fitted using program SURVIV (White 1983). The constraints included $F_{n}=1$ as bears become inactive in the winter, and $p_{d}=1$ because the estimate of $p_{d}$ for models with different relocation rates for live and dead animals is on the boundary. These constraints reduce problems caused by near-singularity while permitting the fidelity rate to be season-specific and the relocation rate to depend on mortality status. Estimates of six-month survival rates, selected standard errors and correlations, and values for Akaike's Information criterion (AIC), are summarized in Table 5. For models 1 and 5, where $p_{l}$ and $p_{d}$ are both estimated, the estimate of $p_{d}$ is 1 . Both models 1 and 5 are close to the singular model $\left(\phi, F, p_{l}, p_{d}, p_{s}\right)$ in Figure 1, and as expected, estimator standard errors and correlations involving $\hat{p}_{d}$ are larger than when a common relocation rate, $p_{r}$, is estimated. (It should be 
Table 5. Models Applied to the Radio Telemetry and Trapping Data, Obtained in 1981 to 1987, From a Study on Black Bears (Sorensen and Powell 1998)

\begin{tabular}{|c|c|c|c|c|c|c|c|}
\hline Model & $\begin{array}{l}\text { Parameters } \\
\text { estimated }\end{array}$ & Constraints & $A / C$ & $\begin{array}{l}\hat{\phi}_{m} \\
(S E) \\
\end{array}$ & $\begin{array}{c}\hat{\phi}_{n} \\
(S E) \\
\end{array}$ & $\begin{array}{c}r\left(\hat{\phi}_{m}, \hat{p}_{d}\right) \\
o r \\
r\left(\hat{\phi}_{m}, \hat{p}_{r}\right)\end{array}$ & $\begin{array}{c}r\left(\hat{\phi}_{n}, \hat{p}_{d}\right) \\
o r \\
r\left(\hat{\phi}_{n}, \hat{p}_{r}\right) \\
\end{array}$ \\
\hline 1 & $\phi_{m}, \phi_{n}, F_{m}, p_{l}, p_{d}, p_{s}$ & $F_{n}=1$ & 99.80 & $\begin{array}{c}0.70 \\
(0.40)\end{array}$ & $\begin{array}{c}0.94 \\
(0.11)\end{array}$ & 0.99 & 0.85 \\
\hline 2 & $\phi_{m}, \phi_{n}, F_{m}, p_{l}, p_{s}$ & $\begin{array}{l}F_{n}=1 \\
p_{d}=1\end{array}$ & 97.80 & $\begin{array}{c}0.70 \\
(0.05)\end{array}$ & $\begin{array}{c}0.94 \\
(0.06)\end{array}$ & & \\
\hline 3 & $\phi_{m}, \phi_{n}, F_{m}, p_{r}, p_{s}$ & $\begin{array}{l}F_{n}=1 \\
p_{l}=p_{d}\end{array}$ & 98.25 & $\begin{array}{c}0.64 \\
(0.06)\end{array}$ & $\begin{array}{c}0.93 \\
(0.06)\end{array}$ & 0.19 & -0.17 \\
\hline 4 & $\phi_{m}, \phi_{n}, F, p_{r}, p_{s}$ & $\begin{array}{l}F_{m}=F_{n} \\
p_{l}=p_{d}\end{array}$ & 98.42 & $\begin{array}{c}0.64 \\
(0.06)\end{array}$ & $\begin{array}{c}0.93 \\
(0.07)\end{array}$ & 0.28 & 0.07 \\
\hline 5 & $\phi, F_{m}, p_{l}, p_{d}, p_{s}$ & $\begin{array}{l}F_{n}=1 \\
\phi_{m}=\phi_{n}\end{array}$ & 107.48 & $\begin{array}{c}0.78 \\
(0.09)\end{array}$ & & 0.90 & \\
\hline 6 & $\phi, F_{m}, p_{r}, p_{s}$ & $\begin{array}{l}F_{n}=1 \\
\phi_{m}=\phi_{n} \\
p_{l}=p_{d}\end{array}$ & 107.50 & $\begin{array}{c}0.75 \\
(0.04)\end{array}$ & & 0.08 & \\
\hline
\end{tabular}

kept in mind that reported standard errors and correlations are approximate because cohort sizes are small.) The AIC values are smaller for models that allow season-specific survival (models 1-4). Models 2, 3, and 4 have the smallest AIC values but differ in that model 2 allows $p_{l}$ and $p_{d}$ to differ. For models 3 and 4 , the common relocation rate is estimated to be 0.79 and 0.77 , respectively, and for model $2, p_{d}$ is 1 and $p_{l}$ is estimated to be 0.78 . Correspondingly, the estimate of annual survival is slightly greater under model 2 than for models 3 and 4 (65\% compared to $60 \%$ and 59\%). The latter estimates agree closely with the estimate of $58 \%$ obtained (assuming common relocation rates) from the telemetry data alone by Sorensen and Powell (1998). Given the necessarily small sample sizes for these large mammals, it is difficult to determine whether relocation rates for live and dead animals are different. However, if relocation rates were close to the values obtained under model 2, where the ratio $p_{l} / p_{d}$ is 0.8 , then the bias expression in Tsai et al. (1999) suggests that survival could be underestimated by almost $10 \%$ if the usual equality assumption is imposed.

\section{DISCUSSION}

The examination of models for the combined analysis of data from a study involving both recapture and relocation by radio telemetry has shown that nonidentifiability can be a serious problem, even in models with a limited number of parameters. The singularity problems arise if different relocation rates are allowed for living and dead animals and the time dependence is the same for the survival rate and the relocation rate for dead animals. 
Analogous results concerning singularity were obtained for a parallel series of models that account for radio failure (Nasution 2000). Investigations for models where emigration does not occur $(F=1)$ have also been conducted. Unlike the models with parameters for both true survival and fidelity, nonidentifiability was not detected (Nasution 2000).

An interesting generalization of our investigations is to telemetry studies where biologists divide animals by cause of death, for example, natural versus fishing mortality (Hightower, Jackson, and Pollock 2001). Analyses of the data have assumed that relocation rates are equal irrespective of cause of death, but if this assumption is violated then estimates of the separate mortality source rates will be biased. Our results suggest that models that attempt to allow different relocation rates by mortality source would likely be unsatisfactory due to nonidentifiability or near nonidentifiability.

Our results are all based on the expected information matrix and its inverse. Sample sizes $\left(R_{i, j}^{r}\right.$ and $\left.R_{i}^{s}\right)$ used in calculations were larger than is usually possible in practice, but large enough for the "large-sample" variances and covariances to be accurate. Problems associated with near-singularity will be worse when sample sizes are smaller than those used here, so our computations should be viewed as optimistic. To describe more accurately the small sample properties of estimators in near-singular models, simulation studies could be conducted using our results as a starting point.

Although we have focused on a combination of recapture and telemetry data, conclusions concerning parameter redundancy and estimator properties will apply to studies where only telemetry data are collected. We strongly recommend that effort to locate dead, as well as living, animals should be consistently high. In many studies, telemetry surveys are conducted as often as weekly or biweekly. For most wildlife populations, reducing the number of surveys per individual and increasing the effort to relocate every animal in each survey will yield data for which appropriate models will have good properties.

\section{ACKNOWLEDGMENTS}

We thank the reviewers and associate editor for comments that led to improving this article.

[Received January 2002. Revised March 2003.]

\section{REFERENCES}

Azzalini, A. (1996), Statistical Inference Based On The Likelihood, New York: Chapman \& Hall.

Bennetts, R. E., Dreitz, V. J., Kitchens, W. M., Hines J. E., and Nichols, J. D. (1999), “Annual Survival of Snail Kites in Florida: Comparisons Between Radio-Telemetry and Capture-Resighting Data,” Auk, 116, 435-447.

Catchpole, E. A., Freeman, S. N., and Morgan, B. J. T. (1996), "Steps to Parameter Redundancy in Age-Dependent Recovery Models,” Journal of Royal Statistical Society, 58, 763-774.

Catchpole, E. A., Kgosi, P. M., and Morgan, B. J. T. (2001), "On the Near-Singularity of Models For Animal Recovery Data," Biometrics, 57, 720-726.

Catchpole, E. A., and Morgan, B. J. T. (1997), “Detecting Parameter Redundancy,” Biometrika, 84, 187-196. 
(2001), "Deficiency of Parameter-Redundant Models," Biometrika, 88, 593-598.

Catchpole, E. A., Morgan, B. J. T., and Freeman, S. N. (1998), "Estimation in Parameter-Redundant Models," Biometrika, 85, 462-468.

Hightower, J. E., Jackson, J. R., and Pollock, K. H. (2001), "Use of Telemetry Methods to Estimate Natural and Fishing Mortality of Striped Bass in Lake Gaston, North Carolina," Transactions of the American Fisheries Society, 130, 557-567.

International Mathematical and Statistical Libraries, Inc. (1987), User's Manual STAT/Library FORTRAN Subroutines for Statistical Analysis, Houston, TX: Author.

Nasution, M. D. (2000), "Estimating Survival from Joint Analysis of Resighting and Radio-Telemetry Data in Wildlife Populations," unpublished doctoral dissertation, North Carolina State University at Raleigh, Dept. of Statistics

Nasution, M. D., Brownie, C., and Pollock, K. H. (2001), "Estimating Survival From Joint Analysis of Resighting and Radio-telemetry Capture-recapture Data For Wild Animals," Journal of Agricultural, Biological, and Environmental Statistics, 6, 461-478.

Pollock, K. H., Bunck, C. M., Winterstein, S. R., and Chen, C. L. (1995), “A Capture-Recapture Survival Analysis Model for Radio Tagged Animals,” Journal of Applied Statistics, 22, 661-672.

Searle, S. R. (1982), Matrix Algebra Useful For Statistics, New York: Wiley.

Sorensen, V. A., and Powell, R. A. (1998), "Estimating Survival Rates of Black Bears," Canadian Journal of Zoology, 76, 1335-1343.

Tsai, K., Pollock, K. H., and Brownie, C. (1999), "Effects of Violation of Assumptions for Survival Analysis methods in Radiotelemetry Studies,'Journal of Wildlife Management, 63, 1369-1375.

Viallefont, A., Lebreton, J. D., Reboulet, A. M., and Gory, G. (1998), "Parameter Identifiability and Model Selection in Capture Recapture Models: A Numerical Approach,” Biometrical Journal, 40, 313-325.

White, G. C. (1983), "Numerical Estimation Of Survival Rates From Band-Recovery and Biotelemetry Data," Journal of Wildlife Management, 47, 716-728. 